\title{
On Colored Edge Cuts in Graphs
}

\author{
Luerbio Faria $^{2}$, Sulamita Klein ${ }^{1}$, Ignasi Sau ${ }^{3}$, Uéverton S. Souza ${ }^{4}$, Rubens Sucupira $^{1,2}$ \\ ${ }^{1}$ Universidade Federal do Rio de Janeiro, PESC/COPPE, Brazil \\ ${ }^{2}$ Universidade do Estado do Rio de Janeiro, Brazil \\ ${ }^{3}$ CNRS, LIRMM, Montpellier, France \\ ${ }^{4}$ Universidade Federal Fluminense, Brazil \\ \{luerbio, sula\}@cos.ufrj.br, \{ignasi.sau, rasucupira\}@gmail.com, \\ uevertonssouza@yahoo.com.br
}

\begin{abstract}
In this work we present some results on the classical and parameterized complexity of finding cuts in edge-colored graphs. In general, we are interested in problems of finding cuts $\{A, B\}$ which minimize or maximize the number of colors occurring in the edges with exactly one endpoint in $A$.
\end{abstract}

\section{Introduction}

A cut in a graph $G$ is a partition of $V(G)$ into two disjoint non-empty sets $A$ and $B$. The cut-set of a cut $\{A, B\}$, denoted by $[A, B]$, is defined as the set of edges of $G$ with exactly one endpoint in $A$. If $s$ and $t$ are two distinct vertices of a graph $G$, an $(s, t)$-cut of $G$ is a cut $\{A, B\}$ of $G$ such that $s \in A$ and $t \in B$.

Motivation. Minimum CUT and Maximum Cut are two of most popular graph problems and they have several applications. An important application of graph edge cuts is image segmentation, in the field of computer vision. Image segmentation can be defined as the task of distinguishing objects from background in images, more precisely, it is the process of partitioning a digital image into multiple segments (sets of pixels, also known as superpixels). Classical methods to obtain image segmentations are based on finding edge cuts in uncolored-edge graphs [Shi and Malik 1997, Felzenszwalb and Huttenlocher 2004]. However, as images can be easily seen as graphs, where pixels' colors can be interpreted as edge's colors, the study of combinatorial problems related to cuts in colored-edge graphs seems to be useful to construct new techniques for image segmentation and for other applications as well.

Let $c: E(G) \rightarrow \mathbb{N}$ be a (not necessarily proper) coloring function defined on the edges of a graph $G$ and let $\{A, B\}$ be a cut in $G$. We denote by $c(A, B)$ the set of colors that appear in the edges of $[A, B]$, that is,

$$
c(A, B)=\{i \in \mathbb{N}: \text { there exists } e \in[A, B] \text { with } c(e)=i\}
$$

Let $\operatorname{im}(c)$ be the image of the coloring function $c: E(G) \rightarrow \mathbb{N}$.

In this paper we consider the following combinatorial problems related to cuts in edge-colored graphs: 


\section{Minimum COLORED CUT}

Input: A connected graph $G$ and a coloring function $c: E(G) \rightarrow \mathbb{N}$. Output: A cut $\{A, B\}$ of $G$ that minimizes $|c(A, B)|$.

Minimum Colored $(s, t)$-CuT

Input: A connected graph $G$, two distinct vertices $s, t \in V(G)$, and a coloring function $c: E(G) \rightarrow \mathbb{N}$.

Output: An $(s, t)$-cut $\{A, B\}$ of $G$ that minimizes $|c(A, B)|$.

\section{MAXIMUM COLORED CUT}

Input: A graph $G$ and a coloring function $c: E(G) \rightarrow \mathbb{N}$.

Output: A cut $\{A, B\}$ of $G$ that maximizes $|c(A, B)|$.

COLORFul Cut

Input: A graph $G$ and a coloring function $c: E(G) \rightarrow \mathbb{N}$.

Output: A cut $\{A, B\}$ of $G$ such that $c(A, B)=\operatorname{im}(c)$, if it exists.

As we can observe, Colorful CUT is a special case of MAXimum Colored Cut, and Minimum Colored Cut are easily Turing-reducible to Minimum ColORED $(s, t)$-CUT.

In this article we focus on the computational complexity of these problems, with special emphasis on their parameterized complexity for several choices of the parameters. For an introduction to the field of Parameterized Complexity, see [Flum and Grohe 2006, Niedermeier 2006, Downey and Fellows 2013, Cygan et al. 2015]. We use standard graph-theoretic notation [Diestel 2010]. Throughout the article, we denote by $n$ the number of vertices of the graph.

\section{Minimum colored cut and colored $(s, t)$-cuts}

To the best of our knowledge, the Minimum Colored Cut and Minimum Colored $(s, t)$-CUT problems were first introduced in [Coudert et al. 2007], using different terminology.

Note that if the coloring function $c$ is injective, that is, if all edges get different colors, then the Minimum Colored Cut and Minimum Colored $(s, t)$-Cut problems correspond exactly to the Minimum CUT and Minimum $(s, t)$-CUT problems, respectively, hence they can be both solved in polynomial time by a classical MAXIMUM FLOW algorithm [Diestel 2010].

\section{Theorem 1.}

- Minimum Colored $(s, t)$-CUT cannot be approximated within a factor of $(1-$ $\epsilon) \ln (c)$ for any constant $\epsilon>0$ unless $\mathrm{P}=\mathrm{NP}$, even if the input graph is bipartite planar graph or complete graph;

- Minimum Colored $(s, t)$-CUT is W[2]-hard parameterized by the cost of the solution, even if the input graph is bipartite planar.

Proof. Reduction from SET COVER which is known to be $\mathrm{W}[2]$-hard when parameterized by the size of the solution [Flum and Grohe 2006] and $(1-\epsilon) \ln (c)$ inapproximable (for any constant $\epsilon>0$ unless $\mathrm{P}=\mathrm{NP}$ ) [Dinur and Steurer 2014].

Theorem 2. Minimum COLORED $(s, t)$-CUT on planar bipartite graphs $G$ remains NPcomplete even when: 
- either, each color of $G$ occurs at most three times and each $(s, t)$-path in $G$ has length two;

- or, each color of $G$ occurs at most twice and each $(s, t)$-path in $G$ has length at most three.

Proof. Reduction from VERTEX COVER on cubic graphs [Blin et al. 2014].

Theorem 3. Minimum Colored $(s, t)$-CuT can be solved in polynomial time when each color appears in at most two $(s, t)$-paths of $G$.

Proof. We can show this using the polynomial algorithm for Monotone WeIGHTED SAT where each variable occurs at most twice [Porschen and Speckenmeyer 2007].

Corollary 4. Minimum Colored $(s, t)$-CUT can be solved in polynomial time when each color occurs at most twice and each $(s, t)$-path has length two.

Theorem 5. Minimum Colored Cut and Minimum Colored $(s, t)$-Cut are FPT when parameterized by the number of colors.

Note that Minimum Colored $(s, t)$-CuT can be solved in polynomial time when $c=O(\log n)$, and can be solved in pseudo-polynomial time when $G$ has an uncolored $(s, t)$-cut of size $O(\log n)$. The following result complements Theorem 5 above.

Lemma 6. Minimum COLORED $(s, t)$-CUT does not admit polynomial kernels when parameterized by the number of colors, unless $\mathrm{NP} \subseteq$ coNP/poly.

Proof. The proof uses OR-composition.

Lemma 7. Minimum COLORED $(s, t)$-CUT are FPT when parameterized by the number of $(s, t)$-paths and the size of the solution.

Lemma 8. Minimum COLORED $(s, t)$-CUT does not admit polynomial kernels when parameterized by the number of $(s, t)$-paths and the size of the solution, unless NP $\subseteq$ $\mathrm{coNP} /$ poly.

Lemma 9. Minimum Colored CUT can be solved in polynomial time when $G$ has an uncolored $(s, t)$-cut of constant size, and it can be solved in $O\left(n^{5}\right)$ time on planar graphs. Theorem 10. Minimum COLORED CUT for directed graphs is NP-hard.

We denote by span of a color $c_{i}$ the number of connected components in the graph induced by the set of edges colored with $c_{i}$.

Lemma 11. Given an edge-colored graph $(G, c)$, let $c_{2}$ denote the number of colors with span at least two. The MINIMUM COLORED CUT problem can be solved in time $3^{c_{2}} \cdot n^{O(1)}$. Lemma 12. Given an edge-colored graph $(G, c)$ and a positive integer $p$, let $c_{p}$ denote the number of colors with span at least $p$. The MINIMUM COLORED CUT problem can be solved by a randomized algorithm in time $3^{c_{p}} \cdot n^{O(1)}$, where the degree of the polynomial depends on $p$.

\section{Maximum colored cut and colorful cut}

In this section we list our results on COLORFul CUT and MAXIMUM Colored CuT. Theorem 13. MAX COLORED CUT remains NP-hard even when restricted to complete graphs.

Theorem 14. MAX COLORED CUT admits a polynomial 2-approximation algorithm. Theorem 15. MAX COLORED CUT can be solved in polynomial time on graphs $G$ colored with a constant number of colors.

Theorem 16. COLORFUl CUT is NP-complete even when each color class induces a clique. 
Theorem 17. COLORFUL CUT can be solved in polynomial time on planar graphs if each color class induces a clique.

Theorem 18. COLORFUL CUT is NP-complete even on planar graphs where each color occurs at most twice and each vertex has degree at most 4.

Corollary 19. COLORFUL CUT is NP-complete, even when restricted to planar graphs with odd cycle transversal number at most 1.

Theorem 20. COLORFUL CUT is NP-complete, even when restricted to graphs with vertex cover number at most 4.

Theorem 21. COlORFUl CUT is NP-complete, even when restricted to planar graphs with feedback vertex set number at most 2.

Theorem 22. MAXIMUM COLORED CUT admits a cubic kernel when parameterized by the number of colors.

Theorem 23. MAXIMUM COLORED CUT admits a cubic kernel when parameterized by the cost of the solution.

\section{References}

Blin, G., Bonizzoni, P., Dondi, R., Rizzi, R., and Sikora, F. (2014). Complexity insights of the minimum duplication problem. Theoretical Computer Science, 530:66 - 79.

Coudert, D., Datta, P., Perennes, S., Rivano, H., and Voge, M. (2007). Shared risk resource group complexity and approximability issues. Parallel Process. Lett., 17(2):169-184.

Cygan, M., Fomin, F. V., Kowalik, L., Lokshtanov, D., Marx, D., Pilipczuk, M., Pilipczuk, M., and Saurabh, S. (2015). Parameterized Algorithms. Springer.

Diestel, R. (2010). Graph Theory, volume 173. Springer-Verlag, 4th edition.

Dinur, I. and Steurer, D. (2014). Analytical approach to parallel repetition. In Proceedings of the 46th Annual ACM Symposium on Theory of Computing, STOC '14, pages 624633, New York, NY, USA. ACM.

Downey, R. G. and Fellows, M. R. (2013). Fundamentals of Parameterized Complexity. Texts in Computer Science. Springer.

Felzenszwalb, P. F. and Huttenlocher, D. P. (2004). Efficient graph-based image segmentation. International Journal of Computer Vision, 59(2):167-181.

Flum, J. and Grohe, M. (2006). Parameterized Complexity Theory. Texts in Theoretical Computer Science. Springer.

Niedermeier, R. (2006). Invitation to Fixed-Parameter Algorithms, volume 31. Oxford University Press.

Porschen, S. and Speckenmeyer, E. (2007). Algorithms for variable-weighted 2-sat and dual problems. In Theory and Applications of Satisfiability Testing-SAT 2007, pages 173-186. Springer.

Shi, J. and Malik, J. (1997). Normalized cuts and image segmentation. In Computer Vision and Pattern Recognition, 1997. Proceedings., 1997 IEEE Computer Society Conference on, pages 731-737. IEEE. 